(one myocardial infarction; one atrial fibrillation). At four hours postadmission, 14 more patients tested positive (13 myocardial infarction and one angina). At 12 hours postadmission, a further seven patients tested positive (five myocardial infarction and two angina).

Of the 19 patients diagnosed as myocardial infarction, a positive troponin $T$ was only present in one patient on admission, and in 13 patients at four hours postadmission. All patients with myocardial infarction had a positive troponin test at 12 hours postadmission.

The TropT slides may be useful at 12 hours to exclude myocardial injury but do not offer improvements in the diagnosis of myocardial infarction in the first hours after presentation at an accident and emergency department

Through the trial we made some observations which should be carefully considered when using TropT slides, especially in areas outside the clinical laboratory. (1) A good light source is required to read the slide, as the positive line can often be faint and the stick needs to be carefully assessed. (2) It is essential to ensure that 20 minutes elapse before reading the slide, as samples with levels of troponin $\mathrm{T}$ close to the sensitivity of the assay only develop after this time. The negative control line often develops within 10 minutes and this could lead to the stick result being misinterpreted in the absence of careful timing. These factors are likely to lead to problems in busy clinical areas, where necessary care with the timing and interpretation of the result may not always occur. (3) Sample handling is also important, and well mixed blood free from haemolysis is required. Since whole blood is used, haemolysis is only apparent on causing a red/brown colouration of the reading area.

We believe that when designing slide tests for use outside the laboratory, manufacturers should ensure that the strength of signal line for a positive result is clear, especially at low levels of the measured analyte. The strength of the negative control line should be equivalent to the signal line obtained by a weakly positive test and should appear after the assay reaction time has been reached to ensure that slides are not read inappropriately early.

In summary, the use of slide tests appears to be very simple but valid results are only obtained with careful and consistent use. The crucial role of the laboratory in the safe use of glucose meters outside of the laboratory is widely accepted. ${ }^{4}$ When clinicians are evaluating slide based tests for use at the patient's bedside they should seek the advise of their laboratory in the choice, evaluation, and training of staff in the use of these systems. Only with such collaboration will such assay systems lead to improvements in patient care.

A TARPEY M LEONARD Departments of Chemical Pathology and *Emergency Medicine, Arrowe Park Hospital, Wirral, Merseyside, L49 5PE

1 Antman EM, Grudzien C, Sacks DB. Evaluation of a rapid bedside assay for detection of serum of a rapid bedside assay for detection of serum

2 Collinson PO, Thomas S, Siu L, et al. Rapid troponin $\mathrm{T}$ measurement in whole blood for the chem 1995;32:454-8.

3 Wu AHB, Valdes R, Apple FS, et al. Cardiac Troponin $T$ immunoassay for the diagnosis of acute myocardial infarction and detection of mino myocardial injury. Clin Chem 1994:40;900-7.

4 Medical Devices Agency Adverse Incident Centre. Extra-laboratory use of blood glucose meters and test strips: contraindications, train ing and advice to users. Safety Notice MDA SN 9616, 1996.

\section{Management of major trauma}

EDITOR,-With major trauma comprising 1 per 1000 emergency cases in Britain there is limited opportunity to develop expertise in the management of this condition. Many of these patients arrive at hospital during unsociable hours when accident and emergency (A\&E) departments are often staffed by inexperienced junior doctors. To provide effective initial resuscitation there should be instant availability of experienced doctors from $A \& E$, anaesthesia, general surgery, and orthopaedic surgery as required, and adequate radiology facilities including 24 hour computerised tomography. Unfortunately many district general hospitals are unable to provide an appropriate service from these specialties. The problem is compounded by the general apathy to trauma shown by many senior surgeons. While we would agree with Leaman ${ }^{1}$ that all hospitals involved in trauma care should submit data to MTOS, we would not expect the results to be encouraging. Recent analysis of data submitted both to MTOS and to the Scottish Trauma Audit Group showed mediocre results, with delays in treatment despite senior staff involvement in initial resuscitation.

It is obvious that Leaman is not a protagonist of aeromedical helicopter transportation; however, in his local region $22 \%$ of the County Air Ambulance missions over the last five years involved interhospital transfers. We suggest this service should continue to be used for transfers involving significant distances, where severe traffic congestion on motorway networks may result in prolonged journeys by land ambulances and for those patients whose clinical condition benefits from this method of transport, for example spinal injuries. The Glasgow Clinical Shock Study Group ${ }^{3}$ provides the gold standard for interhospital transportation; however, as they point out, the development of an integrated transport system involving experienced doctors is unlikely to become a realistic option in Britain due to the financial implications involved between different hospital Trusts.

C MACKAY

Accident and Emergency Department, Selly Oak Hospital, Birmingham K M PORTER

Trauma and Orthopaedics, Selly Oak Hospital, Birmingham

1 Leaman AM. The future for district general hospitals and their A\&E departments. J Accid Emerg Med 1996;13:418-9.

2 Court-Brown CM. Treatment of the polytraumatized patient in the United Kingdom. Clin Orthop Rel Res 1995;318:36-42.

3 Hope A, Runcie CJ. Interhospital transport in the critically ill adult. Br J Intensive Care 1993;3: 187-92.

\section{Treatment of focal status epilepticus with lignocaine}

EDITOR,-We report a patient with status epilepticus with focal seizures following generalised tonic status epilepticus, who was successfully treated with intravenous lignocaine after treatment with diazepam plus phenytoin.

A 68 year old man was admitted with a tonic generalised convulsion. He had a 40 year history of heavy alcohol intake and recurrent minor head traumas, although computerised tomography of the head showed nothing abnormal. Tonic generalised convulsions, each lasting for about one minute, occurred repeatedly. The interval between attacks was about 15 minutes, during which he was unresponsive to verbal stimuli. Diazepam $10 \mathrm{mg}$ was given intravenously with no effect. Phenytoin $750 \mathrm{mg}$ intravenously was then given and the level of consciousness improved within two hours. However, a right tonic hemiconvulsion persisted, although the interval was prolonged to three to four hours on the day after admission. Despite of the additional administration of $1000 \mathrm{mg}$ of phenytoin intravenously over the following 24 hours, the right tonic hemiconvulsion recurred.

An intravenous bolus of $100 \mathrm{mg}$ lignocaine was therefore given during the attack, and the convulsion completely disappeared within about 30 seconds. The patient was then given a continuous intravenous infusion of lignocaine, 1 $\mathrm{mg} / \mathrm{min}$, and oral phenytoin, $300 \mathrm{mg} /$ day. Status epilepticus did not recur. On day 10 , the lignocaine was discontinued and convulsions did not recur thereafter. The electroencephalogram on day 12 revealed no epileptic findings.

Currently, lignocaine is considered as the third line agent in the treatment of status epilepticus when first line agents, such as diazepam and phenytoin, and second line agents, such as barbiturates, fail to control seizures. ${ }^{1}$ However, the best treatment for focal status epilepticus has not yet been established, although barbiturates, carbamazepine, and phenytoin are recommended. ${ }^{2}$ We gave lignocaine because of the ineffectiveness of the first line agents and the possibility of respiratory suppression by second line agents. A search of the literature revealed one case report of focal status epilepticus treated with lignocaine. ${ }^{3}$ This report emphasised that lignocaine does not cause sedation and rarely depresses the cardiovascular or respiratory systems when given in therapeutic dosage. The obvious effectiveness of lignocaine in our case suggests that this is a drug of choice in the treatment of status epilepticus with focal seizures.

H KATO

H KISHIKAWA

S EMURA

T TAKASHIMA K OHMOR

Department of Internal Medicine, Saga Medical School, Saga 849, Fapan H FUJITA

Department of General Medicine, Saga Medical School,

Saga 849, fapan

Teng E, Wilkins P. Lidocaine in status epilepticus. Ann Pharmacother 1994;28:1248-9.

2 Dichter MA. The epilepsies and convulsive disorders. In: J D Wilson et al, eds. Harrison' principles of internal medicine, 12 th ed. New York: McGraw-Hill, 1991:1968-77.

3 Morris HH. Lidocaine: a neglected anticonvulsant? South Med J 1979;72:1564-6.

\section{Decision support for telephone advice}

EDITOR,-We read with interest the paper by Srinivas et al. ${ }^{1}$ Since 1993 we have been researching telephone advice in $\mathrm{A} \& \mathrm{E}$ and general practice and have developed approaches to standardising patient assessment and advice. This includes piloting a telephone consultation skills training package and a computer based decision support system. Our findings, based on an analysis of 340 calls to an A\&E department, concur with those of Srinivas et al, with $23 \%$ advised to attend $\mathrm{A} \& \mathrm{E}, 21 \%$ to attend their GP, and $31 \%$ given home care advice. We found similar support from the nursing staff using the system. We also found that $53 \%$ of callers $(n=81)$ were aware that the nurse was using a computer and the majority $(75 \%)$ believed it to be a good idea to use computers to provide clinical 
decision support. These results provide further evidence of both professional and public support for this approach.

As Srinivas et al point out, the weakness in telephone consultation lies in the area of history taking. It is in this area of patient assessment where decision support is of greatest value. For the record of the consultation to be of most use, particularly if litigation were to arise, it would seem highly desirable to have a complete record of the questions asked and the responses given. Provisional analysis of research that we are undertaking using simulation of calls has identified that nursing staff form a hypothesis as to the nature of the patient's problem early in the consultation.
This is then followed by a process of backward reasoning, gathering evidence to support the hypothesis rather than forward reasoning from symptoms to hypothesis. The approach to the assessment is related to the complexity of the task-the more complex the assessment, the less systematic the approach. ${ }^{3}$ If decision support in telephone consultation is to be of value it should delay the hypothesis generation and promote forward reasoning; it should also be designed to help the user to structure more complex consultations. Decision support systems need to be developed to enable this, while being sufficiently user friendly to be acceptable to staff.
R CROUCH J DALE

Accident and Emergency Department, King's College Hospital, London

1 Srinivas S, Poole F, Redpath J, Underhill TJ. Rrinivas S, Poole F, Redpath J, Underhill TJ. in an A\&E department. J Accid Emerg Med in an A\&E depart

2 Crouch R, Dale J, Patel A, Williams S, Woodley $\mathrm{H}$. Ringing the changes: developing, piloting and evaluating a telephone advice system in accident $\&$ emergency and general practice setings. London: Departments of A\&E Medicine and General Practice. King's College School of Medicine and Dentistry, 1996.

3 Corcoran SA. Task complexity and nursing expertise as factors in decision making. Nursing Res 1986;35:107-12.

\section{Correction}

An uncorrected scanning error occurred in the title of the abstract "Prehospital diazepam: an audit of use" in the last issue (volume 14, page 126), where "adult" was printed instead of "audit". Also the name of one of the authors (H Cosgrove) was omitted. The authors have informed us of an error in their analyses, which means that the data presented are incorrect, though the conclusions are unaltered. We understand that they will be submitting a definitive paper on this subject to the journal. This will be peer reviewed in the usual way.

\section{Trauma Care (UK) 1st Biennial Conference "Total Trauma Care" \\ Harrogate International Centre 25-27 November 1997}

This conference and future conferences will promote integration of trauma care by establishing "Total Trauma Care". The conference will provide access to relevant advances and current thinking in all aspects of trauma. Trauma care professionals are all aware of the advances being made in their respective areas of involvement with the trauma victim and the conference will recognise those parts of the chain of care which, often repeatedly, break down. The conference will allow both local and national professional groups to work together on an equal basis, through a common doctrine, to a single goal. Professionals attending the conference can then understand the whole chain of care concept, with a view to reducing morbidity and mortality associated with trauma.

Details available from Total Trauma Care, c/o Conference Secretariat, Index Communications Meeting Services, Crown House, 28 Winchester Road, Romsey, Hampshire SO51 8AA. Tel: 01794 511331/511332; fax: 01794 511455 . 